\title{
Erratum to: Association between the functional ITGAM rs1143679 G/A polymorphism and systemic lupus erythematosus/ lupus nephritis or rheumatoid arthritis: an update meta-analysis
}

\author{
Young Ho Lee $\cdot$ Sang-Cheol Bae
}

Published online: 24 December 2014

(C) Springer-Verlag Berlin Heidelberg 2014

\section{Erratum to: Rheumatol Int}

DOI 10.1007/s00296-014-3156-2

It has been brought to the authors' attention that some of the data originally presented in DOI 10.1007/s00296-0143156-2 are not accurately reflective of one of the original studies cited.

\section{On Page 4 Paragraph 2, the article states}

"However, one Latin American study showed no evidence of association between the A allele and LN in Latin Americans (OR 1.440, $95 \%$ CI 0.961, 2.157, $p=0.077$; Table 3; Fig. 3)."

Correction: The study referenced [Ref: 6] does indeed shows evidence of an association between the A allele and LN in Latin Americans. Furthermore, the data presented here do not accurately reflect that which was presented in the original study, which reads OR 2.58, $95 \%$ CI 1.65 , $4.03, p<0.01$. The text should also refer to Table 3 and Fig. 4a.

\section{On Page 4 Paragraph 3, the article states}

"Meta-analysis showed no evidence of association between the A allele and RA, either in the overall study population or in Europeans (OR 1.078, 95 \% CI 0.967, 1.191, $p=0.177$; OR 1.064, $95 \%$ CI $0.950,1.191, p=0.287$; Table 3; Fig. 4)."

Correction: this paragraph should refer to Fig. $4 \mathrm{~b}$.

On Page 5 Fig. 2, the figure reads

"Study name: Toller-Kawahisa et al. 2013."

Correction: This should read Toller-Kawahisa et al. 2014. A corrected figure is included on the next page.

The online version of the original article can be found under doi:10.1007/s00296-014-3156-2.

\section{Y. H. Lee $(\square)$}

Division of Rheumatology, Department of Internal Medicine,

Korea University Anam Hospital, Korea University College

of Medicine, 126-1, Anam-dong 5-ga, Seongbuk-gu,

Seoul 136-705, Korea

e-mail: lyhcgh@korea.ac.kr

S.-C. Bae

The Hospital for Rheumatic Diseases, Hanyang University

Medical Center, Seoul, Korea 


\begin{tabular}{|c|c|c|c|c|c|c|c|c|c|}
\hline \multirow[t]{2}{*}{ Study name } & \multirow[t]{2}{*}{ Ethnicity } & \multicolumn{4}{|c|}{ Statistics for each study } & \multicolumn{4}{|c|}{ Odds ratio and $95 \% \mathrm{Cl}$} \\
\hline & & $\begin{array}{l}\text { Odds } \\
\text { ratio }\end{array}$ & $\begin{array}{l}\text { Lower } \\
\text { limit }\end{array}$ & $\begin{array}{c}\text { Upper } \\
\text { limit }\end{array}$ & p-Value & & & & \\
\hline Toller-Kawahisa et al, 2014 & Latin American & 1.798 & 1.195 & 2.705 & 0.005 & & & & I \\
\hline Jarvinen et al, 2010 & European & 1.595 & 1.196 & 2.127 & 0.001 & & & & \\
\hline Warchol et al, 2011 & European & 1.763 & 1.194 & 2.602 & 0.004 & & & & \\
\hline Suarez-Gestal-1 et al, 2009 & European & 1.418 & 0.872 & 2.305 & 0.159 & & & & \\
\hline Suarez-Gestal-2 et al, 2009 & European & 1.953 & 1.581 & 2.413 & 0.000 & & & & \\
\hline Suarez-Gestal-3 et al, 2009 & European & 1.607 & 1.216 & 2.122 & 0.001 & & & & \\
\hline Suarez-Gestal-4 et al, 2009 & European & 1.589 & 1.019 & 2.477 & 0.041 & & & & \\
\hline Suarez-Gestal-5 et al, 2009 & European & 1.823 & 0.993 & 3.346 & 0.053 & & & & \\
\hline Suarez-Gestal-6 et al, 2009 & European & 1.561 & 1.105 & 2.205 & 0.011 & & & & \\
\hline Suarez-Gestal-7 et al, 2009 & European & 1.100 & 0.579 & 2.092 & 0.771 & & & & \\
\hline Suarez-Gestal-8 et al, 2009 & European & 1.234 & 0.677 & 2.250 & 0.492 & & & & \\
\hline Suarez-Gestal et al-9, 2009 & European & 2.569 & 1.374 & 4.803 & 0.003 & & & & \\
\hline Yang-1 et al, 2009 & Asian & 2.230 & 1.146 & 4.336 & 0.018 & & & & \\
\hline Yang-2 et al, 2009 & Asian & 3.149 & 1.725 & 5.749 & 0.000 & & & & 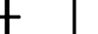 \\
\hline Han-1 et al, 2009 & Latin American & 2.066 & 1.452 & 2.940 & 0.000 & & & & \\
\hline Han-2 et al, 2009 & European & 2.123 & 1.609 & 2.799 & 0.000 & & & & \\
\hline Han-3 et al, 2009 & Latin American & 2.014 & 1.441 & 2.814 & 0.000 & & & & \\
\hline Han-4 et al, 2009 & Latin American & 1.571 & 1.077 & 2.294 & 0.019 & & & & \\
\hline Nath-1 et al, 2008 & European & 1.782 & 1.559 & 2.037 & 0.000 & & & & \\
\hline Nath-2 et al, 2008 & African & 1.521 & 1.205 & 1.921 & 0.000 & & & & \\
\hline \multirow{4}{*}{ Nath-3 et al, 2008} & Gullah & 2.070 & 1.273 & 3.364 & 0.003 & & & & \\
\hline & & 1.773 & 1.655 & 1.901 & 0.000 & & & $\|$ & \\
\hline & & & & & & 0.1 & 0.5 & 2 & 10 \\
\hline & & & & & & & Control & SLE & \\
\hline
\end{tabular}

Fig. 2 ORs and $95 \%$ CIs for individual studies and pooled data, for the association between the A allele of the ITGAM rs 1143679 A/G polymorphism and SLE, in all study subjects

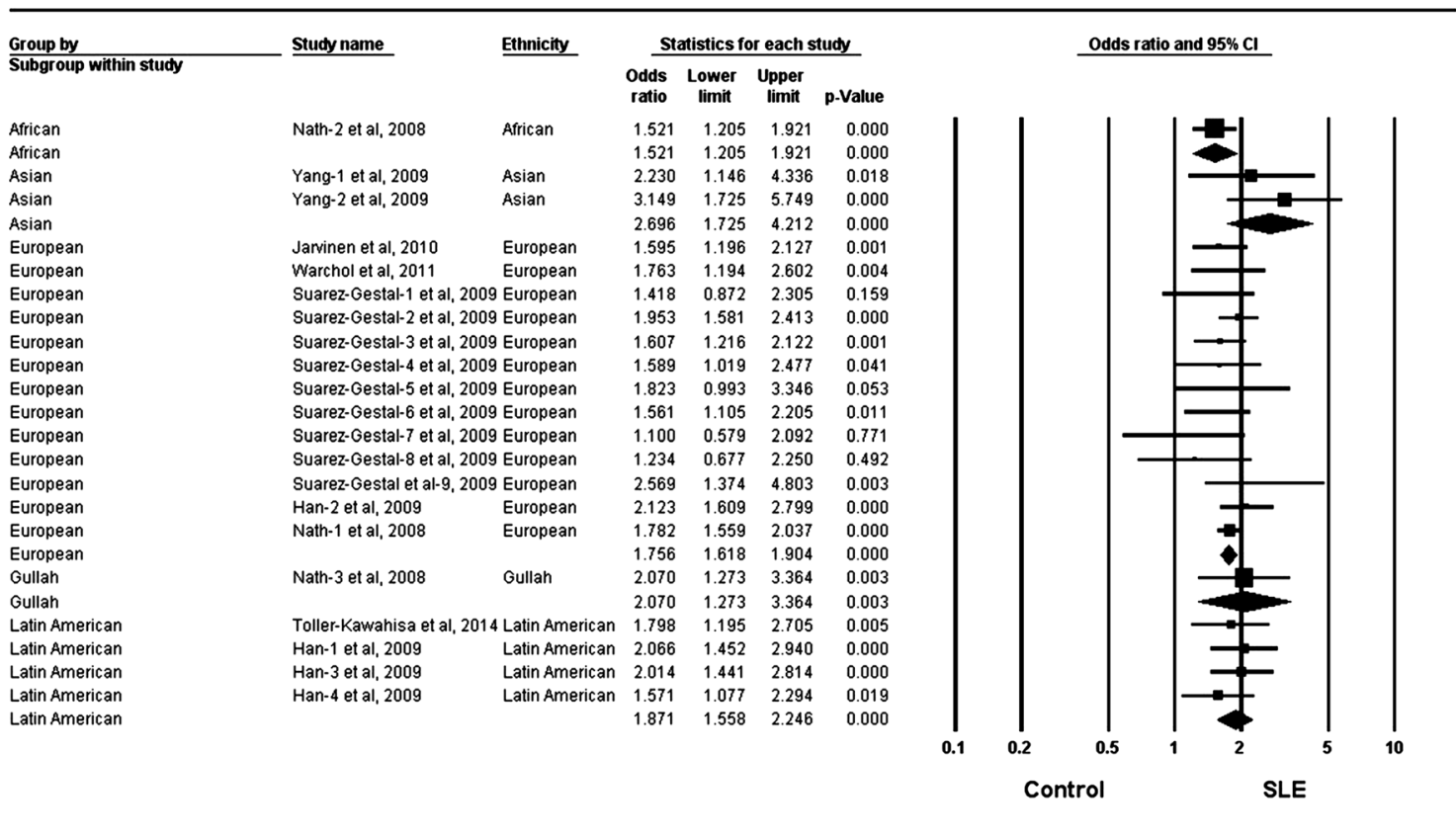

Fig. 3 ORs and $95 \%$ CIs for individual studies and pooled data, for the association between the A allele of the ITGAM rs 1143679 A/G polymorphism and SLE, in each ethnic group

On Page 6 Fig. 3, the figure reads

"Study name: Toller-Kawahisa et al. 2013."
Correction: This should read Toller-Kawahisa et al. 2014. A corrected figure is included above. 


\section{On Page 6 Table 3, the table reads}

"LN A versus G allele, Latin American population, OR $1.440,95 \%$ CI $0.961,2.157, p=0.077 . "$

Correction: The table does not accurately reflect the data presented in the original study [Ref: 6]. The table should instead read as: LN A versus $G$ allele, Latin American population, OR 2.58, $95 \%$ CI 1.65, 4.03, $p<0.01$. A corrected table is included below.
On Page 7 Fig. 4a, the figure reads

"Toller-Kawahisa et al. 2013, Latin American ethnicity, OR $1.440,95 \%$ CI $0.961,2.157, p=0.077$.”

Correction: The figure does not accurately reflect the data presented in the original study [Ref: 6]. The figure should instead read as: Toller-Kawahisa et al. 2014, Latin American ethnicity, OR 2.58, $95 \%$ CI 1.65, 4.03, $p<0.01$. A corrected figure is included below.

Table 3 Meta-analysis of the association between the ITGAM rs 1143679 G/A polymorphism and LN or RA

\begin{tabular}{|c|c|c|c|c|c|c|c|c|}
\hline \multirow[t]{2}{*}{ Disease/polymorphism } & \multirow[t]{2}{*}{ Population } & \multirow[t]{2}{*}{ No. of studies } & \multicolumn{3}{|c|}{ Test of association } & \multicolumn{3}{|c|}{ Test of heterogeneity } \\
\hline & & & OR & $95 \% \mathrm{CI}$ & $p$ & Model & $I^{2}$ & $p$ \\
\hline \multirow[t]{3}{*}{ LN A versus $G$ allele } & Overall & 3 & 1.844 & $1.443-2.357$ & $1.0 \times 10^{-7}$ & $\mathrm{~F}$ & 45.4 & 0.160 \\
\hline & European & 2 & 2.131 & $1.565-2.903$ & $1.6 \times 10^{-7}$ & $\mathrm{~F}$ & 27.5 & 0.240 \\
\hline & Latin American & 1 & 2.580 & $1.650-4.030$ & $<0.01$ & NA & NA & NA \\
\hline \multirow[t]{3}{*}{ RA A versus $G$ allele } & Overall & 3 & 1.078 & $0.967-1.191$ & 0.177 & $\mathrm{~F}$ & 0 & 0.489 \\
\hline & European & 2 & 1.064 & $0.950-1.191$ & 0.287 & $\mathrm{~F}$ & 0 & 0.369 \\
\hline & Latin American & 1 & 1.247 & $0.855-1.820$ & 0.252 & NA & NA & NA \\
\hline
\end{tabular}

$F$ fixed-effect model, $R$ random-effects model, $N A$ not available

Fig. 4 ORs and $95 \%$ CIs for individual studies and pooled data, for the association between the A allele of the ITGAM rs $1143679 \mathrm{~A} / \mathrm{G}$ polymorphism and LN (a) or RA (b), in all study subjects
$\underline{\mathbf{A}}$

\begin{tabular}{|c|c|c|c|c|c|c|c|c|c|c|}
\hline \multirow[t]{2}{*}{ Study name } & \multirow[t]{2}{*}{ Ethnicity } & \multicolumn{4}{|c|}{ Statistics for each study } & \multicolumn{5}{|c|}{ Odds ratio and $95 \% \mathrm{Cl}$} \\
\hline & & $\begin{array}{c}\text { Odds } \\
\text { ratio }\end{array}$ & $\begin{array}{c}\text { Lower } \\
\text { limit }\end{array}$ & $\begin{array}{c}\text { Upper } \\
\text { limit }\end{array}$ & p-Value & & & & & \\
\hline Toller-Kawahisa ef al, 2014 & Latin American & 2.588 & 1.659 & 4.039 & $<0.01$ & & & & & \\
\hline Jarvinen et al, 2010 & European & 1.786 & 1.165 & 2.738 & 0.008 & & & & & \\
\hline \multirow[t]{4}{*}{ Warchol et al, 2011} & European & 2.587 & 1.654 & 4.047 & 0.000 & & & & & \\
\hline & & 2.270 & 1.761 & 2.926 & 0.000 & & & & & \\
\hline & & & & & & 0.1 & 0.2 & 0.5 & 1 & 10 \\
\hline & & & & & & & Cor & & LN & \\
\hline
\end{tabular}

